Hewitt, L. F. (1952). J. gen. Microbiol. 7, 362-371.

\title{
Diphtheria Bacteriophages and their Relation to the Development of Bacterial Variants
}

\author{
By L. F. HEWITT \\ Serum Research Institute (Medical Research Council), \\ Carshalton, Surrey
}

SUMMARY: Many strains of Corynebacterium diphtheriae carry phages capable of lysing other strains. Diphtheria phages are type-specific and do not lyse strains which are serologically unrelated but susceptibility to a phage may vary among different members of the same serological type and this can be used to differentiate subtypes of different biological behaviour. Phage-resistant mutants are readily selected in diphtheria cultures and the resistant mutants frequently have characteristics quite distinct from those of the parent strain, e.g. virulent organisms have been isolated from a number of avirulent strains. It is suggested that the possibility of converting avirulent strains to virulent ones may be of importance epidemiologically and that the phage selection of mutants may account for the wide variety of serological types isolated from patients. Some strains found to be resistant to type-specific phages have been shown to be lysogenic and when subjected to ultraviolet irradiation liberate phage particles which are capable of lysing susceptible strains of the same serological type. The diphtheria phages investigated are thermolabile, difficult to filter, of fairly large size and give rise to small plaques of lysis with susceptible strains.

Strains of Corynebacterium diphtheriae have been classified into some sixty serological types by agglutination methods (Hewitt, 1947, 1948a), and the literature detailing the variations in virulence, colonial form, fermentation reactions, etc., is too vast for brief review, but the origin of these wide variations and their effect on the epidemiology of diphtheria remain obscure. It is found that antibiotics such as penicillin and streptomycin alter the characteristic properties of diphtheria strains by the selection of resistant mutants (Hewitt, 1948b), but these drugs are unlikely to have played any important part in the evolution of the strains encountered in infections. One purpose of this communication is to investigate the possibility that bacteriophages may be the natural agents responsible for the great variety of strains that are isolated from diphtheria patients and carriers.

Phage typing for the identification of infecting strains has become an established procedure in the case of a number of organisms (Burnet, 1930; Craigie \& Yen, 1938; Felix \& Anderson, 1951; Wilson \& Atkinson, 1945; Boyd, 1950), but although the occurrence of phages active against $C$. diphtheriae has been described by a number of workers (Blair, 1924; Smith \& Jordan, 1931; Stone \& Hobby, 1934; Keogh, Simmons \& Anderson, 1938; Toshach, 1950; Freeman, 1951; Parsons \& Frobisher, 1951; Freeman \& Morse, 1952) there is insufficient evidence available to judge whether $C$. diphtheriae can be classified on the basis of phage sensitivity or whether diphtheria bacteriophages are of 
common occurrence. An investigation into the natural distribution of diphtheria bacteriophages, their properties and specificity is therefore necessary.

\section{MATERIALS AND METHODS}

Sources of strains. The strains of $C$. diphtheriae used were in some cases freshly isolated from patients in various parts of the world (Great Britain, continental Europe, the Middle East, Africa, Australia and America), and others which had been kept in laboratory cultivation. Methods of isolation have been described previously (Hewitt, 1947).

Serological typing. Anderson, Happold, McLeod \& Thomson's (1931) heated blood tellurite agar medium was used for the characterization of the Mitis, Intermedius and Gravis groups, and serological typing was by agglutination with rabbit sera as described previously (Hewitt, 1947, 1948a).

Virulence and toxigenicity tests. The virulence of strains was tested by the lethal effect of cultures washed off Loeffler slopes and injected subcutaneously into guinea-pigs. The toxigenicity was determined by growing the strains in a standardized papain digest broth and titrating the toxin in the filtrate by a modification of the Römer (1909) intracutaneous test as described previously (Hewitt, 1948a).

Isolation of phages. The phages investigated were isolated from phagecarrying diphtheria strains. As described in a later section ultra-violet irradiation was used to demonstrate the lysogenicity of some strains, but in other cases it was possible to detect lysis when a culture was 'spotted' on an agar plate already inoculated with another strain, as found by Fisk (1942) in the case of staphylococcus phages. For the isolation of some phages mixed cultures of two diphtheria strains in broth were incubated and the filtrate was added to a culture of the strain for which the phage was sought. Several repetitions of this process were generally necessary before appreciable phage activity could be detected.

Phage titrations. The lysis of broth cultures was frequently a poor guide to phage activity, but areas of lysis on solid media proved a convenient index of phage potency. Broth cultures of the susceptible strain were streaked on nutrient agar plates and loopfuls of suitable phage dilutions were spotted on the streaks. Dilutions were made in broth in order to avoid variation in the concentration of cations or other broth constituents. Enumeration of phage particles by the counting of plaques on solid media by the method of Gratia (1936) is not practicable with many diphtheria strains, since growth is not confluent but tends to occur in clumps which obscure the plaques.

\section{RESULTS}

Phage-carrying strains. Of the first eight diphtheria strains examined at random five readily yielded phages. Two of the phage-carrying strains belonged to the Mitis group, one was Gravis and the other two Intermedius. The phages were, as shown later, highly specific in their range of activity, but phages of widely different host specificity can be isolated from a single 
lysogenic strain. Thus from one lysogenic Intermedius strain three phage filtrates were obtained by subculturing in the presence of a Gravis type I strain, a Mitis type $\mathbf{X}$ strain and an Intermedius strain respectively. The phages obtained each lysed only the homologous strain and other strains of the same serological type. This, however, does not necessarily mean that the lysogenic strain was carrying a number of different phages but may have been due to adaptation of the phage to the susceptible strain during the course of isolation.

Adaptation of phage. When a phage for any particular serological type was required it was obtained either by culturing together a strain of this type with a phage-carrying strain, or by adapting one of the phages already obtained to the new type. This adaptation was effected by adding phage to a broth culture of the strain, incubating for $18 \mathrm{hr}$. and adding the supernatant fluid to a fresh subculture of the strain. If no effective phage had appeared after six subcultures the experiment was generally abandoned and a fresh combination of phage and strain was investigated in the same way. By this method thirty-six bacteriophages effective against twenty-four serological types have been obtained, and more than eighty strains have been found to be lysed by one or other of the phages.

Heat lability of phage. Diphtheria phages were completely destroyed by heating at $55^{\circ}$ for $30 \mathrm{~min}$. but survived at $50^{\circ}$; C. diphtheriae cultures were sterilized by heating at $50^{\circ}$ for $30 \mathrm{~min}$. but survived at $46^{\circ}$. Using this difference in heat stability it is possible to rid phage suspensions of living diphtheria bacilli by first centrifuging and then heating at $50^{\circ}$, but chance bacterial contaminants introduced during centrifuging operations, etc., may survive heating at $50^{\circ}$, and filtration yields a clearer product.

Filtrability of phage. Filtration of phage through a Seitz or Ford sterilizing pad or a Pasteur-Chamberland L3 candle removed lytic activity. Sterilization without serious loss of activity was effected, however, by filtration through Gradocol membranes of average pore diameter (A.P.D.) between 0.5 and $0.7 \mu$.

Size of phage particles. The plaques produced by diphtheria phages are small, generally not exceeding $0.3 \mathrm{~mm}$. in diameter. Elford \& Andrewes (1932) point out that the formation of small plaques is indicative of large phage particles, and this is in accord with the observations mentioned in the previous section that phage activity is readily lost on filtration. Using Gradocol membranes of different porosity it was found that some activity passed through membranes of $0 \cdot 115 \mu$. A.P.D. but all lytic activity was held back by membranes of $0.075 \mu$ A.P.D., the lytic activity being recovered by washing the membranes with broth. This evidence suggests a particle size of about $60-80 \mathrm{~m} \mu$.; the phages examined by Elford $\&$ Andrewes (1932) ranged in size from the smallest of 8-12 $\mathrm{m} \mu$. to the largest of 50-75 $\mathrm{m} \mu$. It appears, therefore, that diphtheria phages are of comparatively large size, and further work is being carried out to define the particle size more closely.

Relation of phage-susceptibility to serological specificity. Thirty-six phages have been investigated, and these have been found to be type-specific, each 
being active only against strains of a single serological type; but, as shown below, the degree of specificity of phage-susceptibility is higher than that of serological specificity and in some cases the phages revealed differences between strains of the same serological type. So far, phages are available for twenty-four different serological types, and a few examples are quoted to illustrate the degrees of specificity observed.

In the case of Gravis type I strains five phages have been prepared. These phages lysed a number of Gravis type I strains but no Mitis or Intermedius strains, nor any Gravis strains which were not serologically related. The phage titres were not, however, the same when tested against different Gravis type I strains, some of which showed varying degrees of resistance to one or other of the phages. Results with three contrasting Gravis type I strains are shown in Table 1.

Table 1. Titres of phage activities against different Gravis type I strains

\begin{tabular}{cccc} 
& \multicolumn{3}{c}{ Strain } \\
Phage & A & B & C \\
1 & 100 & 1000 & 10 \\
2 & 1000 & 100 & 0 \\
3 & 100 & 0 & 1 \\
4 & 1000 & 1 & 1 \\
5 & 1000 & 10 & 1
\end{tabular}

Titres are expressed in terms of the dilution of phage of which one loopful produced confluent lysis on a culture of the strain on an agar plate.

The explanation of these differences in susceptibility to phage among members of a single serological type is discussed in a later section where the lysogenicity of strain $\mathrm{C}$ is demonstrated.

In the case of a Gravis type II phage only the homologous strain was lysed. When adapted by subculture with another Gravis II strain its range of activity was widened, so that it lysed six Gravis II strains, but as some strains of this type were still not susceptible it was further adapted to one of the non-susceptible strains; this further adaptation decreased the lytic activity against some of the previously susceptible strains. Thus the phages are typespecific but further degrees of specificity are shown in the activity of some phages. This is clearly seen in the case of phages prepared against a Mitis type III strain.

All Mitis type III strains are agglutinated by the same agglutinating rabbit serum prepared by intravenous injection of a killed vaccine, but of twentyfour strains examined, only twelve were susceptible to the type-specific phage, the others being completely resistant. The two subtypes were quite clearly differentiated, the resistant strains being virulent and toxigenic, whilst the susceptible strains were found to be avirulent and non-toxigenic. Further implications of this phage differentiation of subtypes become apparent in the next section dealing with phage-resistant mutants.

Selection of phage-resistant mutants. Addition of the appropriate phage to broth cultures of susceptible diphtheria bacilli has in no case been found to 
produce complete clearing but, after incubation, resistant mutants grow out and these on subculture are found to be no longer susceptible to the phage. The phage-resistant mutants, however, sometimes differ from the parent strain in respects other than that of phage-susceptibility. Thus in the case of a Gravis type VIII strain and the appropriate phage, the phage-resistant culture was virulent and toxigenic, whilst the original strain was avirulent.

An interesting example of this difference in properties is in the case of Mitis type III strains. As mentioned in the previous section, the phagesusceptible strains are avirulent. Phage-resistant mutants isolated from six of these strains proved to be virulent and toxigenic. Through the agency of phage, therefore, the avirulent strains had been converted to the virulent subtype.

Heterogeneity of resistant mutants. It is noteworthy that the virulent phageresistant mutants selected by the action of bacteriophage on the avirulent Mitis III strains described in the previous section were not all of equal toxigenicity. The titre of toxin produced in the standardized papain digest broth varied in the case of the different strains from 0.05 to 4.0 units $/ \mathrm{ml}$. Moreover, in each case by picking colonies on solid media, phage-resistant mutants remaining avirulent and non-toxigenic could be selected. Differences in the properties of phage-resistant mutants were also found in the case of phage-resistant mutants of a Gravis type I strain (strain A in Table 1). In one experiment phage was added to a culture of the strain on an agar plate, and, after incubation, an area of confluent lysis appeared where the phage had been added. There were, however, a few colonies of resistant mutants. Five of these were picked off with a platinum wire and subcultured separately. Apart from differences in growth characters, granularity of growth, etc., the toxigenicity of the mutants under standardized conditions varied from 0.01 to $\mathbf{0 . 2} \mathrm{unit} / \mathrm{ml}$. It becomes clear, therefore, that all the mutants selected by means of their resistance are not identical.

Further evidence of this is seen in the degree of resistance of different mutants to various phages. Examples of these differences among wild strains are given in Table 1, and it is now possible to consider the properties of phage-resistant mutants obtained in the laboratory. Again working with the Gravis type I, strain A, mutants were prepared against each of the five Gravis type I phages previously mentioned, and the susceptibility of each resistant mutant to the same phages was tested. The results are summarized in Table 2. Resistant strains are designated by the phage to which they were made resistant, e.g. $\mathrm{R} 1$ signifies the mutant resistant to phage no. 1 .

The mutants $R 1$ and $R 3$ which were still susceptible to phage 2 were now subjected to the action of this phage, and the resistant mutants $R 1,2$ and R3, 2 were isolated, but these were found to be resistant to all five phages. The experiments summarized in Table 2 show that it is possible to produce in the laboratory strains of varying phage susceptibility similar to those isolated from diphtheria patients and carriers.

Lysogenicity and ultra-violet irradiation. It has just been shown that it is possible to produce in the laboratory mutant strains of varying phage suscepti- 
bility, and it seemed possible that the similar range of susceptibility encountered among strains in the field might reflect the phage history of such strains. Further evidence was sought, therefore, that diphtheria strains showing resistance to type specific phages had encountered phages at some stage in their history.

Evidence of this was found in the case of Gravis type I strains. Strain A, as mentioned previously, is susceptible to lysis by five type-specific phages, whilst strains $\mathbf{C}$ and $\mathbf{D}$ show varying degrees of resistance. Cultures of strain $\mathbf{A}$

Table 2. Susceptibility of resistant mutants to other phages

\begin{tabular}{crrrcc} 
Phage & \multicolumn{5}{c}{ Strain no. } \\
no. & R1 & R2 & R3 & R4 & R5 \\
1 & 0 & 100 & 0 & 0 & 0 \\
2 & 100 & 0 & 100 & 0 & 0 \\
3 & 0 & 100 & 0 & 0 & 0 \\
4 & 0 & 100 & 100 & 0 & 0 \\
5 & 100 & 100 & 100 & 0 & 0
\end{tabular}

Titres are given in terms of the dilution of the phage producing confluent lysis.

were streaked on agar plates and strains $\mathrm{C}$ and $\mathrm{D}$ were spotted on these streaks. When the plates were incubated little evidence of lysis was seen, but if the plates were first subjected to ultra-violet irradiation definite evidence of lysogenicity was obtained. The induction of phage liberation by ultra-violet irradiation has been described in the case of Bacillus megaterium by Lwoff, Siminovitch \& Kjeldegaard (1950). It was found necessary to choose the dosage carefully, since too intense an exposure to ultra-violet light either sterilizes the cultures or results in so high a mortality rate that, instead of confluent growth of the susceptible strain, there are separate colonies obscuring areas of lysis. Under the experimental conditions used the appropriate exposure was for $10 \mathrm{sec}$. at a distance of $30 \mathrm{~cm}$. from a mercury vapour lamp, but an attempt was not made to calibrate the intensity of radiation in terms of energy units. When the plates which had been irradiated under these conditions were incubated areas of lysis were seen where the phageresistant strains had been applied. A portion of the lysed area of growth was transferred to a broth culture of the susceptible strain with a platinum wire. After incubation the supernatant fluid was filtered through a Gradocol membrane and repetition of the passage yielded a potent phage filtrate which lysed twenty-six other Gravis type I strains. It should be noted that the phage did not lyse the lysogenic strain from which it had been isolated, nor did it lyse two other resistant strains. Thus the strains resistant to the typespecific phage had been shown to be carriers of a phage active against susceptible strains of the same serological type. Direct evidence of previous contact with phage is therefore available. 


\section{DISCUSSION}

The hypothesis on which this work is based is that, in the case of diphtheria at any rate, bacteriophage may well be one of the principal natural agents responsible for controlling bacterial variation in infections. The means by which bacteria suddenly increase in virulence and epidemics occur is rather obscure for although, as with other living organisms, mutation among the bacteria has long been recognized, it has not been clear how whole cultures could rapidly undergo a permanent change in their characters. Adaptive changes are generally temporary and dependent upon environment, whilst the occurrence of one mutant bacterium in perhaps a million would not be expected to affect appreciably the properties of the culture as a whole, and the processes of natural selection would appear to be too slow to account for the rapid alterations encountered in infections. On the other hand, bacteriophage will lyse all the normal bacteria in a susceptible culture leaving intact only the few mutants resistant to the phage so that the resistant organisms that grow out will consist solely of mutants. Thus in a few hours the entire bacterial population changes from that of the parent strain to the mutant form. It thus seems at least plausible that bacteriophage may be of importance in epidemiology.

It was first necessary to establish that diphtheria phages occur naturally, and this was readily shown. The first eight strains examined at random were of six serological types, and five of these, including Gravis, Intermedius and Mitis strains, were found to be phage carriers, and potent phage filtrates have been obtained from each of them. Exhaustive surveys covering many strains from different sources would be necessary to give a statistically significant estimate of the frequency of occurrence of diphtheria phages, but it is already clear that they are of quite common occurrence.

It was found that the phages are type-specific and do not lyse strains which are not serologically related. The specificity is of a higher order than that seen in agglutination reactions, and many strains show varying degrees of resistance to their type-specific phages. There are several points of interest in this phage resistance. In the first place it was found that strains of one serological type, Mitis type III, were clearly differentiated into two subtypes according to their phage susceptibility. The phage-resistant strains were found to be virulent and toxigenic, whilst the susceptible strains were avirulent and nontoxigenic. Moreover, when some of the phage-susceptible avirulent strains were subjected to the action of phage and the phage-resistant mutants which grew out were isolated, they were found to be virulent and toxigenic. It had thus been possible in the laboratory to convert one subtype into the other and to transmute an avirulent into a virulent strain. Apart from the theoretical interest of these results the possible epidemiological significance is quite evident. In addition to providing an explanation of the variation in virulence of infecting organisms the experiment indicates how the carrier of an apparently harmless organism might become the disseminator of disease should an appropriate phage be present. At the present time the incidence 
of diphtheria in this country is unprecedentedly low and a high proportion of the child population has been rendered immune to diphtheria toxin, but there are still many carriers of the diphtheria bacillus. A strain received recently was isolated from a patient with sore throat and transient pyrexia. The child was in an orphanage in which the whole community had been fully immunized. The organism appeared to be typically $C$. diphtheriae but was quite avirulent and might well be dismissed as of no epidemiological significance. If, however, such strains in contact with phage can be transformed into virulent organisms they may prove the latent source of epidemics in the future, should the present trend of decline in immunization rate result in a susceptible population. It is of interest in this connexion to note the report of Edward \& Allison (1951) that of forty-four strains of C. diphtheriae isolated from fully immunized persons nine were avirulent, and recently Bradshaw, Dixon, Mawson, Turner \& Zinnemann (1952) reported the isolation of five avirulent strains of $C$. diphtheriae from school children in a highly immunized community. That this alteration of virulence in the presence of phage is not a fortuitous and rare phenomenon is suggested by the recent work of Freeman (1951), Parsons \& Frobisher (1951) and Freeman \& Morse (1952), who also report the conversion of avirulent strains to virulence after treatment with bacteriophage.

It is of interest to decide if there is any evidence that diphtheria strains isolated from infected patients have actually been subjected to the action of bacteriophage at any stage of their history, and whether phage has played any part in the evolution of the large number of serological types of $C$. diphtheriae encountered in field work. Indirect evidence is available in the varying phage-resistance of strains isolated from diphtheria patients and carriers. The case of Gravis type I strains can be taken as an example. Several typespecific Gravis type I phages of different origin have been isolated, and it has been found that the susceptibility of different Gravis type I strains to these phages varies widely. It seemed reasonable to conclude that the phageresistant strains had developed resistance by means of mutant selection through contact with the appropriate bacteriophage at some stage of their history, and it remained necessary to show that such contact with bacteriophage had in fact occurred. The necessary evidence was forthcoming when it was found that ultra-violet irradiation induced the liberation from two phageresistant Gravis type I strains of phage particles which lysed susceptible strains of the same serological type. The resistant strains could only have acquired such lysogenicity by previous contact with type-specific phage.

Of course, the fact that it may not be possible to demonstrate lysogenicity in all phage-resistant strains does not exclude the possibility that they have acquired such resistance after contact with bacteriophage, since not all phage-resistant mutants are lysogenic. Mutants with a slightly different surface structure fail to absorb phage and are not lysed. This second method of developing phage resistance may well be of great importance in the evolution of new serological types of bacteria, since only organisms differing from the rest of the culture will survive the lytic action of phage. Perhaps it should 
be emphasized that not all the phage-resistant mutants growing out after phage has acted on a strain are identical. Although similar in their resistance to phage the mutants obtained from a single strain may vary widely in other respects. For example, some of the phage-resistant mutants obtained by the action of phage on an avirulent strain were virulent whilst others remained avirulent but differed from the parent strain in their phage resistance and growth characters.

Reverting to the original working hypothesis that bacteriophage may be of significance in controlling bacterial variation in infections, the evidence so far collected indicates that, in the case of diphtheria, phages occur naturally, that they are able to effect rapid and significant changes in the characters of strains and that many strains encountered in infections present evidence of having been in contact with phage at some stage of their history. It is concluded, therefore, that the data available offer sufficient encouragement to continue the investigation.

\section{REFERENCES}

Anderson, J. S., Happold, F. C., Mcleod, J. W. \& Thomson, J. G. (1931). On the existence of two forms of diphtheria bacillus-B. diphtheriae gravis and B. diphtheriae mitis - and a new medium for their differentiation and for the bacteriological diagnosis of diphtheria. J. Path. Bact. 34, 667.

BLAIR, J. E. (1924). A lytic principle (bacteriophage) for Corynebacterium diphtheriae. $J$. infect. Dis. 35, 401.

Boyd, J. S. K. (1950). The symbiotic bacteriophages of Salmonella typhi-murium. J. Path. Bact. 62, 501.

Bradshaw, D. B., Dixon, C. W., Mawson, F. M., Turner, G. H. \& Zinnemann, K. S. (1952). Diphtheria carrier investigation. Lancet, i, 558.

BunNet, F. M. (1930). Bacteriophage activity and the antigenic structure of bacteria. J. Path. Bact. 33, 647.

Craigie, J. \& Yen, C. H. (1938). The demonstration of types of B. typhosus by means of preparations of Type II Vi phage. Canad. publ. Hlth J. 29, 448.

Edward, D. G. FF. \& Allison, V. D. (1951). Diphtheria in the immunized with observations on a diphtheria-like disease associated with non-toxigenic strains of Corynebacterium diphtheriae. J. Hyg., Camb. 49, 205.

Elford, W. J. \& ANDrewEs, C. H. (1932). The sizes of different bacteriophages. Brit. J. exp. Path. 13, 446.

Felix, A. \& Anderson, E. S. (1951). Bacteriophage, virulence and agglutination tests with a strain of Salmonella typhi of low virulence. J. Hyg., Camb. 49, 349.

Fisk, R. T. (1942). Studies on staphylococci. I. Occurrence of bacteriophage carriers among strains of Staphylococcus aureus. J. infect. Dis. 71, 153.

Freeman, V. J. (1951). Studies on the virulence of bacteriophage infected strains of Corynebacterium diphtheriae. J. Bact. 61, 675.

Freeman, V. J. \& Morse, I. U. (1952). Further observations on the change to virulence of bacteriophage-infected avirulent strains of Corynebacterium diphtheriae. J. Bact. 63, 407.

Gratia, A. (1936). Des relations numériques entre bactéries lysogènes et particulier de bactériophage. C.R. Soc. Biol., Paris, 122, 812.

Hewitt, L. F. (1947). Serological typing of C. diphtheriae. Brit. J. exp. Path. 28, 338.

Hewitr, L. F. (1948a). Virulence and toxigenicity of different serological types of C. diphtheriae. Brit. J. exp. Path. 29, 181. 
Hewrte, L. F. (1948b). Protection by streptomycin, penicillin and licheniformin against C. diphtheriae infections. Brit. J. exp. Path. 29, 289.

Keogit, E. V., Simmons, R. T. \& Andersox, E. (1938). Type-specific bacteriophages for C. diphtheriae. J. Path. Bact. 46, 565.

Lwoff, A., Siminovitch, L. \& KJeldegaAnd, N. (1950). Induction de la production de bactériophages chez une bactérie lysogène. Ann. Inst. Pasteur, 79, 815.

Parsons, E. I. \& Frobisher, M. (1951). Effect of bacteriophage on virulence of Corynebacterium diphtheriae. Proc. Soc. exp. Biol., N.Y. 78, 746.

Römer, P. H. (1909). Über den Nachweis sehr kleiner Mengen des Diphtheriegiftes. Z. ImmunForsch. 3, 208.

Sмith, G. H. \& Jordan, E. F. (1931). Bacillus diphtheriae in its relationship to bacteriophage. J. Bact. $21,75$.

Stone, F. M. \& Hoввy, G. L. (1934). A coccoid form of $C$. diphtheriae susceptible to bacteriophage. J. Bact. 27, 403.

Tosinach, S. (1950). Bacteriophages for C. diphtheriae. Canad. J. publ. Hlth, 41, 332.

Wilson, G. S. \& Atkinson, J. D. (1945). Typing of staphylococci by the bacteriophage method. Lancet, i, 647 .

(Received 8 May 1952) 